\title{
Índices bioclimáticos para a cultura de café
}

\author{
H udson de P. Carvalho ${ }^{1}$, Benjamim de Melo', Paulo G. Rabelo', \\ Cláudio R. da Silva ${ }^{1} \&$ Reginaldo de Camargo ${ }^{1}$
}

\begin{abstract}
RESU MO
Além dos graus-dia, vắrios são os sistemas de unidades bioclimáticas, embora sua utilização e limitação ainda sejam pouco conhecidas na cafeicultura. D iante disto, este trabal ho objetivou avaliar o desempenho de quatro índices bioclimáticos para estimativa da duração dos subperíodos florescimento-fruto verde cana, fruto verde cana -fruto cereja e florescimento-fruto cereja, na cultura de café. Durante o ano agrícola 2008/2009 foram avaliados índice térmico, acúmulo da radiação fotossinteticamente ativa, índice heliotérmico de Geslin e acúmulo da evapotranspiração de referência, em dez cultivares de café plantadas em U berlândia, M G. O s dados obtidos para cada índice bioclimático foram analisados através de estatística clássica, obtendo-se a média, o desvio padrão e o coeficiente de variação, em que este último parâmetro serviu de base para a escolha do índice bioclimático utilizado na quantificação da duração dos subperíodos de cada cultivar de café e na classificação do ciclo em precoce, médio e tardio. Verificou-se que os índices heliotérmico de Geslin e acúmulo da evapotranspiração de referência, apresentaram os menores coeficientes de variação médios entre as cultivares.
\end{abstract}

Palavras-chave: Coffea arabica, evapotranspiração, índice heliotérmico de G eslin, índice térmico, radiação fotossinteticamente ativa

\section{Bioclimatic indices for the coffee crop}

\begin{abstract}
Besides degree-days, which considers air temperature, there are several bioclimatic unit systems. How ever, their use and limitation is little known for coffee crop. Therefore, this study evaluated the performance of four bioclimatic indices to estimate the length of the sub-periods flow ering-light green fruit, light green fruit-cherry fruit and flowering-cherry fruit, in coffee crop. The thermal index, accumulation of photosynthetic active radiation, Geslin's heliothermic index and the accumulation of the reference evapotranspiration were evaluated during the agricultural year 2008/2009 for ten coffee cultivars, in U berlândia-M G, Brazil. The data for each bioclimatic index were analyzed with the classical statistics, determining the mean, the standard error, and the coefficient of variation. This last parameter was the basis for the choice of the bioclimatic index used for the quantification of the length of the sub-periods for each coffee cultivar and for the classification of the cycle as early, medium and late. Geslin's heliothermic index and accumulation of the reference evapotranspiration presented the smallest mean coefficients of variation among the cultivars.
\end{abstract}

Key words: Coffea arabica, evapotranspiration, G eslin's heliothermic index, thermal index, photosynthetic active radiation 


\section{INTRODUÇÃO}

A cafeicultura brasileira tem, ao seu dispor, diversas cultivares com características produtivas e épocas de maturação diferenciadas. Contudo, essas cultivares foram classificadas, quanto à época de maturação dos frutos, em condições edafoclimáticas dos locais onde foram selecionadas e se sabe que, apesar da precocidade de maturação dos frutos ser controlada geneticamente, é bastante afetada por condições edafoclimáticas regionais e microclimáticas (Petek et al., 2009). Portanto, podem haver comportamentos diferenciados entre as cultivares de café quanto às diferenças edafoclimáticas entre regiões de cultivo e anos distintos. Como consequência, pode acontecer de os diferenciais em maturação dos frutos esperados não se concretizarem. Cultivares caracterizadas como épocas de maturação diferentes nos seus locais de origem, podem tornar-se iguais em outros locais, devido à interação entre genótipos e ambientes.

A interação entre as culturas e os fatores ambientais representados pelos diversos elementos meteorológicos, afeta o crescimento e o desenvolvimento sob diversas maneiras nos diferentes estádios de uma cultura (Camargo et al., 1986).

A temperatura do ar é um dos elementos comumente envolvidos em estudos de relação clima-planta, pois influencia os processos fisiológicos das plantas, interferindo em cada subperíodo de seu ciclo (Brunini et al., 1976). Dentre as ferramentas disponíveis para se caracterizar esta interferência, o sistema de unidades térmicas ou graus-dia é um dos mais utilizados.

Diversos autores utilizaram os graus-dia ou índice térmico para relacionar o desenvolvimento das plantas com a temperatura ambiente (Lima \& Silva, 2008, Pezzopane et al., 2008). Apesar de bastante utilizada, deve-se mencionar que o conceito de unidades térmicas recebe algumas críticas (Xue et al., 2004). Entre elas está o fato de que existem vários métodos de cálculo, o que pode ser uma limitação para comparar os graus-dia das fases de desenvolvimento das cultivares em diferentes trabalhos. Algumas vezes também os relatos nos trabalhos não são claros quanto ao método de cálculo utilizado (McMaster \& Wilhelm, 1997).

Outra fonte de crítica se refere às temperaturas basais, geralmente consideradas constantes ao longo do ciclo da cultura o que, muitas vezes, também não é uma pressuposição adequada tendo em vista que essas temperaturas não são constantes durante o ciclo de desenvolvimento vegetal para muitas cultivares (Pereira et al., 2002).

Além do índice térmico vários são os sistemas de unidades bioclimáticas possíveis de serem usados para a estimativa da duração do ciclo de culturas, mas a utilização e a limitação de cada um deles são pouco relatadas na literatura, principalmente para a cultura de café, em que as informações citadas dizem respeito apenas ao índice térmico.

Visando identificar as limitações de diferentes índices bioclimáticos na cultura do trigo, Sastry \& Chakravarty (1982), avaliaram cinco deles e concluíram que o acúmulo da evapotranspiração potencial e da radiação fotossinteticamente ativa mostrou menor variabilidade, respectivamente para os subperíodos germinação-antese e germinação-maturação.
Nessa linha de trabalho Sentelhas \& Ungaro (1998) avaliaram cinco índices bioclimáticos para a estimativa da duração do ciclo de duas cultivares e de um híbrido de girassol e constataram que o índice térmico apresentou a menor variação ao longo das diferentes épocas de semeadura avaliadas.

O objetivo deste trabalho foi avaliar o desempenho de quatro índices bioclimáticos para estimativa da duração do ciclo de cultivares de café, nas condições climatológicas de Uberlândia, Estado de Minas Gerais.

\section{MATERIAL E MÉTODOS}

O experimento foi desenvolvido em Uberlândia, MG, na latitude $18^{\circ} 56^{\prime} 45^{\prime}$ ' S, longitude 45 ${ }^{\circ}$ 52' 23" 'W e altitude de 912 m. Os elementos meteorológicos radiação solar global, temperatura do ar, umidade relativa do ar e velocidade do vento, foram coletados por uma estação climatológica automática da rede de observação meteorológica do Instituto Nacional de Meteorologia, em escala horária, no período de 01/09/2008 a 31/05/2009.

Os cafeeiros (Coffea arabica L.) foram irrigados por gotejamento e fertirrigados com nível de adubação, para obtenção de altas produtividades. O controle de pragas, doenças e plantas daninhas foi realizado dentro dos padrões agronômicos recomendados para a cultura de café. O plantio ocorreu em 12 de janeiro de 2000, no espaçamento de 3,5 m entre linhas e $0,7 \mathrm{~m}$ entre plantas na linha de plantio.

O delineamento experimental foi o de blocos casualizados com quatro repetições e as parcelas compostas por oito plantas, considerando-se nas avaliações somente as quatro plantas centrais (parcela útil). Os tratamentos se referem às variedades de cafeeiros estudadas: Icatu Precoce IAC 3282 (considerado padrão); Acaiá Cerrado MG 1474; Catuaí Vermelho IAC 15; Catuaí Vermelho IAC 99; Catuaí Amarelo IAC 17; Catuaí Amarelo IAC 62; Catuaí Vermelho IAC 144; Mundo Novo IAC 379-19; Rubi MG 1192 e Topázio MG 1190.

Durante o ano agrícola 2008/2009 observou-se a data de ocorrência das fases fenológicas de florescimento (florada mais expressiva), fruto verde cana (pelo menos $85 \%$ dos frutos da planta com coloração verde cana) e fruto cereja (pelo menos $85 \%$ dos frutos da planta na condição de fruto cereja), em cada planta da parcela útil sendo, portanto, analisado um universo de 16 plantas de cada cultivar.

Com a data de ocorrência das fases fenológicas de cada planta e com os dados climatológicos medidos durante os subperíodos (florescimento até a fase de fruto verde cana, fruto verde cana até a fase de fruto cereja e ciclo total - que equivale ao período compreendido entre o florescimento e a fase de fruto cereja), pôde-se calcular os seguintes índices bioclimáticos:

a) Índice Térmico(IT):

$$
\mathrm{IT}=\sum_{\mathrm{i}=1}^{\mathrm{J}}\left(\mathrm{T} \mathrm{m}_{\mathrm{i}}-\mathrm{Tb}\right) \cdot \mathrm{Pt}
$$


em que: IT é dado em ${ }^{\circ} \mathrm{C}$ dia; $\mathrm{Tm}_{\mathrm{i}}$ é a temperatura média do ar do iésimo dia (obtida pela média de 24 leituras), em ${ }^{\circ} \mathrm{C}$; Tb é a temperatura basal inferior para a cultura de café, igual a $12{ }^{\circ} \mathrm{C}$, sendo este uma aproximação entre o valor relatado por Lima \& Silva (2008) e Pezzopane et al. (2008); Pt é o período de tempo correspondente a 1 dia; i é o índice para cada dia de cada subperíodo e J representa o número de dias de cada subperíodo.

b) Índice Heliotérmico de Geslin (IHG):

$$
\mathrm{IHG}=\frac{1}{100} \cdot \sum_{\mathrm{i}=1}^{\mathrm{J}}\left(\mathrm{Tm}_{\mathrm{i}} \cdot \mathrm{N}_{\mathrm{i}}\right)
$$

em que: $\mathrm{IHG}$ é dado em ${ }^{\circ} \mathrm{C}$ hora e $\mathrm{N}_{\mathrm{i}}$ é o fotoperíodo do iésimo dia, em horas.

c) Acúmulo da Evapotranspiração de Referência (AETR):

$$
\operatorname{AETR}=\sum_{\mathrm{i}=1}^{\mathrm{J}} \mathrm{ETo}_{\mathrm{i}}
$$

em que: ETo é a evapotranspiração de referência do iésimo dia estimada pelo método de Penman-Monteith, em mm. Assim como ETo, os valores de radiação líquida necessários ao cálculo da evapotranspiração por este método, foram estimados de acordo com Allen et al. (1998).

d) Acúmulo da Radiação Fotossinteticamente Ativa (ARFA):

$$
\mathrm{ARFA}=\sum_{\mathrm{i}=1}^{\mathrm{J}} \mathrm{RFA}_{\mathrm{i}}
$$

em que: ARFA é dado em $M J m^{-2}$ dia $^{-1}$; RFA é a radiação fotossinteticamente ativa do iésimo dia, estimada pela relação RFA $=0,44 *$ Qg (Assunção, 1994) e Qg é a radiação solar global ( $\left.\mathrm{MJ} \mathrm{m}^{-2} \mathrm{dia}^{-1}\right)$ medida na estação meteorológica.
Os dados obtidos para cada índice bioclimático em cada planta das diferentes cultivares, foram analisados através de estatística clássica, obtendo-se a média, o desvio padrão e o coeficiente de variação; este último parâmetro serviu de base para a escolha do índice bioclimático a ser utilizado na quantificação da duração dos subperíodos de desenvolvimento dos frutos das cultivares. Uma vez escolhido o índice bioclimático, foram realizadas a análise de variância e a aplicação do teste de Scott \& Knott para diferenciar os grupos de cultivares quanto à duração do ciclo total em precoce, intermediária e tardia, tomando-se como padrão a cultivar Icatu Precoce IAC 3282.

\section{RESULTADOS E DISCUSSÃO}

Para o período entre o florescimento pleno e a fase de fruto verde cana, o índice que apresentou a menor variabilidade foi o acúmulo da evapotranspiração de referência (AETR), seguido dos índices heliotérmico de Geslin (IHG), acúmulo da radiação fotossinteticamente ativa (ARFA) e térmico (IT), conforme apresentado na Tabela 1. Resultado semelhante observaram Sastry \& Chakravarty (1982) para a cultura do trigo, ao verificarem que o índice baseado no acúmulo da evapotranspiração potencial apresentou uma das menores variâncias para os subperíodos germinação-antese e germinação-maturação.

As cultivares Catuaí Amarelo IAC 17, Catuaí Amarelo IAC 62, Catuaí Vermelho IAC 144 e Mundo Novo 379-19, não apresentaram variação entre os índices avaliados no subperíodo de florescimento a fruto verde cana. Este resultado aconteceu porque a fase de fruto verde cana foi alcançada na mesma data pelas plantas dessas cultivares impossibilitando, portanto, a diferenciação na análise estatística realizada. Os índices avaliados não apresentaram bons resultados para a cultivar Catuaí Vermelho IAC 99, indicando que a duração desse período de desenvolvimento esteja sendo influenciada por outro elemento ou fator meteorológico, não considerado neste trabalho.

Os resultados de variância dos índices estatísticos avaliados para o subperíodo compreendido entre a fase de fruto verde cana e a fase de fruto cereja, estão compilados na Tabela 2;

\begin{tabular}{|c|c|c|c|c|c|c|}
\hline \multirow{2}{*}{ Cultivar } & \multicolumn{2}{|c|}{ Data ou subperíodo de ocorrência } & \multicolumn{4}{|c|}{ Coeficiente de variação* (\%) } \\
\hline & Florescimento & Fruto verde cana & IT & AETR & IHG & ARFA \\
\hline Icatu Precoce IAC 3282 (padrão) & 23/09/2008 & $13 / 03$ a 20/03/2009 & 1,9 & 1,8 & 1,9 & 2,0 \\
\hline Acaiá Cerrado MG 1474 & $23 / 09 / 2008$ & $06 / 03$ a $13 / 03 / 2009$ & 2,1 & 1,9 & 2,0 & 2,1 \\
\hline Catuaí Vermelho IAC 15 & $23 / 09 / 2008$ & $13 / 03$ a $27 / 04 / 2009$ & 3,4 & 3,1 & 3,3 & 3,8 \\
\hline Catuaí Vermelho IAC 99 & $23 / 09 / 2008$ & $06 / 03$ a $27 / 04 / 2009$ & 13,6 & 11,7 & 13,1 & 13,1 \\
\hline Catuaí Amarelo IAC 17 & $23 / 09 / 2008$ & $13 / 04 / 2009$ & 0,0 & 0,0 & 0,0 & 0,0 \\
\hline Catuaí Amarelo IAC 62 & $23 / 09 / 2008$ & $13 / 04 / 2009$ & 0,0 & 0,0 & 0,0 & 0,0 \\
\hline Catuaí Vermelho IAC 144 & $23 / 09 / 2008$ & $13 / 04 / 2009$ & 0,0 & 0,0 & 0,0 & 0,0 \\
\hline Mundo Novo IAC 379-19 & $23 / 09 / 2008$ & $06 / 03 / 2009$ & 0,0 & 0,0 & 0,0 & 0,0 \\
\hline Rubi MG 1192 & $23 / 09 / 2008$ & $13 / 04$ a 20/04/2009 & 1,5 & 1,3 & 1,5 & 1,6 \\
\hline Topázio MG 1190 & $23 / 09 / 2008$ & $20 / 03$ a 20/04/2009 & 6,3 & 5,2 & 6,1 & 5,3 \\
\hline Média & & & 2,9 & 2,5 & 2,8 & 2,8 \\
\hline
\end{tabular}

Tabela 1. Coeficientes de variação calculados para diferentes índices bioclimáticos da cultura de café (Coffea arabica L.), durante o subperíodo do florescimento até o fruto verde cana

* IT - Índice térmico, AETR - Acúmulo de evapotranspiração de referência, IHG - Índice heliotérmico de Geslin, ARFA - Acúmulo da radiação fotossinteticamente ativa 
Tabela 2. Coeficientes de variação calculados para diferentes índices bioclimáticos da cultura de café (Coffea arabica L.), durante o subperíodo de frutos verde cana a fruto cereja

\begin{tabular}{|c|c|c|c|c|c|c|}
\hline \multirow{2}{*}{ Cultivar } & \multicolumn{2}{|c|}{ Data ou subperíodo de ocorrência } & \multicolumn{4}{|c|}{ Coeficiente de variação* (\%) } \\
\hline & Fruto verde cana & Fruto cereja & IT & AETR & IHG & ARFA \\
\hline Icatu Precoce IAC 3282 (padrão) & $13 / 03$ a 20/03/2009 & $13 / 04 / 2009$ & 1,2 & 2,1 & 1,1 & 0,9 \\
\hline Acaiá Cerrado MG 1474 & 06/03 a 13/03/2009 & $13 / 04 / 2009$ & 10,4 & 11,3 & 9,8 & 11,1 \\
\hline Catuaí Vermelho IAC 15 & $13 / 03$ a $27 / 04 / 2009$ & $27 / 04$ a $26 / 05 / 2009$ & 27,3 & 26,9 & 2,2 & 27,6 \\
\hline Catuaí Vermelho IAC 99 & $06 / 03$ a $27 / 04 / 2009$ & $13 / 04$ a $26 / 05 / 2009$ & 41,6 & 40,3 & 41,0 & 39,6 \\
\hline Catuaí Amarelo IAC 17 & $13 / 04 / 2009$ & $12 / 05$ a $26 / 05 / 2009$ & 20,8 & 23,2 & 21,2 & 22,5 \\
\hline Catuaí Amarelo IAC 62 & $13 / 04 / 2009$ & $26 / 05 / 2009$ & 0,0 & 0,0 & 0,0 & 0,0 \\
\hline Catuaí Vermelho IAC 144 & $13 / 04 / 2009$ & $12 / 05$ a $26 / 05 / 2009$ & 16,5 & 18,2 & 16,8 & 17,8 \\
\hline Mundo Novo IAC 379-19 & $06 / 03 / 2009$ & $27 / 04 / 2009$ & 0,0 & 0,0 & 0,0 & 0,0 \\
\hline Rubi MG 1192 & $13 / 04$ a 20/04/2009 & $12 / 05$ a $26 / 05 / 2009$ & 16,8 & 18,3 & 17,1 & 17,8 \\
\hline Topázio MG 1190 & $20 / 03$ a 20/04/2009 & $12 / 05$ a $26 / 05 / 2009$ & 16,0 & 19,2 & 16,6 & 21,6 \\
\hline Média & & & 15,1 & 15,9 & 12,6 & 15,9 \\
\hline
\end{tabular}

* IT - Índice térmico, AETR - Acúmulo de evapotranspiração de referência, IHG - Índice heliotérmico de Geslin, ARFA - Acúmulo da radiação fotossinteticamente ativa

analisando-a, nota-se que as plantas das cultivares Catuaí Amarelo IAC 62 e Mundo Novo IAC 379-19, não apresentaram variação durante esta fase, o que impediu a diferenciação na análise de variância; apesar disto, para a cultivar Catuaí Vermelho IAC 15 o IHG apresentou menor variância em relação aos demais índices testados e mostrou que na fase compreendida entre fruto verde cana e fruto cereja, esta cultivar é sensível à interação entre a temperatura do ar e o fotoperíodo.

Com relação à cultivar Icatu Precoce IAC 3282, o índice ARFA apresentou menor coeficiente de variação porém este valor está próximo dos valores encontrados para IHG e IT; para as demais cultivares os índices bioclimáticos avaliados indicaram coeficientes de variação altos, sinal de que na fase de desenvolvimento entre fruto verde cana e fruto cereja, eles não são adequados para a estimativa da referida fase e que essas plantas, no referido período, não são sensíveis aos elementos e fatores meteorológicos que compõem esses índices; contudo, quando se avalia a média obtida para cada índice nota-se que o IHG apresentou o menor coeficiente de variação, podendo ser usado com maior segurança quando comparado com os demais índices, para a estimativa da duração dessa fase em outras cultivares de café, diferentes daquelas avaliadas neste trabalho.

Os coeficientes de variação para os índices bioclimáticos analisados obtidos para o ciclo total, estão contidos na Tabela 3; analisando-a, verifica-se que, para as cultivares Acaiá
Cerrado MG 1474, Catuaí Amarelo IAC 62 e Mundo Novo IAC 379-19, não foi possível verificar qual dos índices apresentou menor coeficiente de variação, uma vez que não houve variação entre as plantas analisadas mas, quando foi calculada a média dos coeficientes de variação, observou-se pequena diferença entre os índices, além de que os valores médios calculados foram baixos. Não obstante, o IHG e o AETR apresentaram resultados semelhantes, porém inferiores aos obtidos pelo IT e ARFA. Sentelhas \& Ungaro (1998) verificaram, para a cultura do girassol, que os coeficientes de variação calculados para IT e IHG apresentaram valores próximos e baixos, como encontrado neste trabalho, mas os autores constataram que o primeiro apresentou melhor resultado que o segundo.

Comumente, o índice térmico é o mais usado para a estimativa da duração do ciclo de culturas. Apesar de não ter apresentado a menor variabilidade para a cultura de café, o IT mostra facilidades para seu uso, dado à menor quantidade de elementos meteorológicos necessários para sua determinação. Não obstante, este resultado sofreu influência da temperatura base inferior, visto que o valor utilizado neste trabalho pode ter contribuído para aumentar a variância.

A partir do resultado contido na Tabela 3, foi realizada análise estatística para verificar a diferença estatística entre a duração do ciclo das cultivares de café. Neste estudo foram utilizados os dados obtidos pelos índices AETR (Figura 1A) e IHG (Figura 1B), os quais apresentaram os menores coeficientes de variação

Tabela 3. Coeficientes de variação calculados para diferentes índices bioclimáticos da cultura de café (Coffea arabica L.), durante o subperíodo do florescimento a fruto cereja

\begin{tabular}{|c|c|c|c|c|c|c|}
\hline \multirow{2}{*}{ Cultivar } & \multicolumn{2}{|c|}{ Data ou subperíodo de ocorrência } & \multicolumn{4}{|c|}{ Coeficiente de variação* (\%) } \\
\hline & Florescimento & Fruto cereja & IT & AETR & IHG & ARFA \\
\hline Icatu Precoce IAC 3282 (padrão) & 23/09/2008 & $13 / 04 / 2009$ & 1,5 & 1,3 & 1,5 & 1,6 \\
\hline Acaiá Cerrado MG 1474 & $23 / 09 / 2008$ & $13 / 04 / 2009$ & 0,0 & 0,0 & 0,0 & 0,0 \\
\hline Catuaí Vermelho IAC 15 & $23 / 09 / 2008$ & $27 / 04$ a $26 / 05 / 2009$ & 4,8 & 4,4 & 2,8 & 5,4 \\
\hline Catuaí Vermelho IAC 99 & 23/09/2008 & $13 / 04$ a $26 / 05 / 2009$ & 6,6 & 6,1 & 6,6 & 7,5 \\
\hline Catuaí Amarelo IAC 17 & $23 / 09 / 2008$ & $12 / 05$ a $26 / 05 / 2009$ & 2,7 & 2,7 & 2,7 & 3,2 \\
\hline Catuaí Amarelo IAC 62 & 23/09/2008 & $26 / 05 / 2009$ & 0,0 & 0,0 & 0,0 & 0,0 \\
\hline Catuaí Vermelho IAC 144 & $23 / 09 / 2008$ & $12 / 05$ a 26/05/2009 & 2,3 & 2,3 & 2,3 & 2,7 \\
\hline Mundo Novo IAC 379-19 & $23 / 09 / 2008$ & $27 / 04 / 2009$ & 0,0 & 0,0 & 0,0 & 0,0 \\
\hline Rubi MG 1192 & $23 / 09 / 2008$ & $12 / 05$ a 26/05/2009 & 2,3 & 2,3 & 2,3 & 2,7 \\
\hline Topázio MG 1190 & $23 / 09 / 2008$ & $12 / 05$ a 26/05/2009 & 6,4 & 5,9 & 6,3 & 7,4 \\
\hline Média & & & 2,7 & 2,5 & 2,5 & 3,1 \\
\hline
\end{tabular}

* IT - Índice térmico, AETR - Acúmulo de evapotranspiração de referência, IHG - Índice heliotérmico de Geslin, ARFA - Acúmulo da radiação fotossinteticamente ativa 
para o ciclo total e um dos menores para a fase de florescimento e fruto verde cana. Analisando essas figuras notou-se que as cultivares foram divididas pela análise estatística, em relação ao ciclo total (florescimento a fruto cereja), em três grupos de maturação. Nesta avaliação foi utilizada, como padrão de precocidade, a cultivar Icatu Precoce IAC 3282. A cultivar Acaiá Cerrado MG 1474 apresentou-se tão precoce quanto a cultivar padrão diferenciando-se, porém, da Mundo Novo IAC 379-19, classificada pelo teste estatístico como de ciclo intermediário.

A.

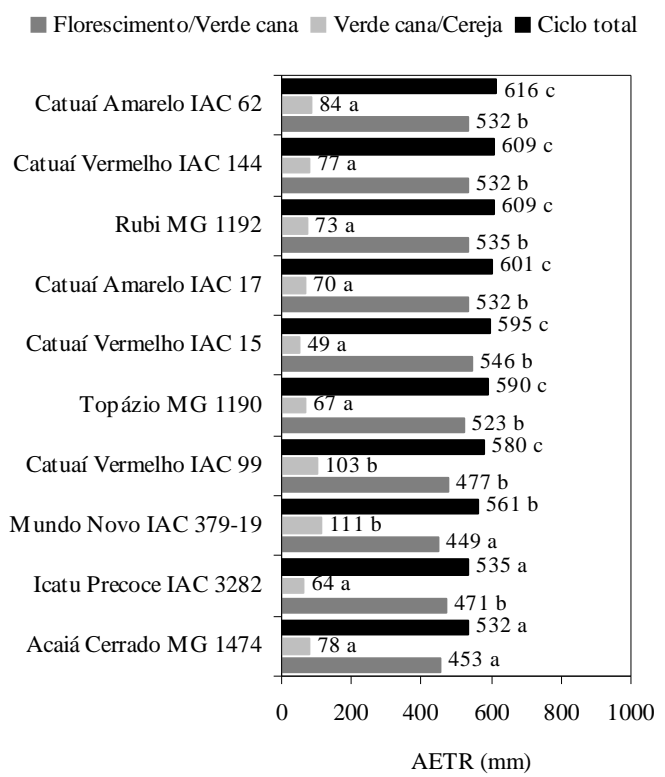

B.

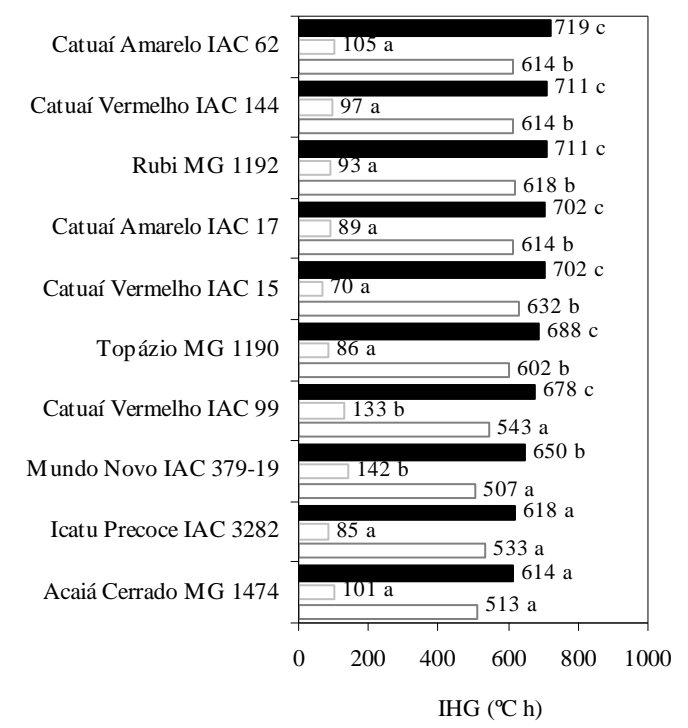

Figura 1. Valores médios do acúmulo da evapotranspiração de referência (AETR) (A) e do índice heliotérmico de Geslin (IHG) (B) para os subperíodos compreendidosentre o florescimento até a fase de fruto verde cana (florescimento/verde cana), fase de fruto verde cana até a fase de fruto cereja (verde cana/cereja) e florescimento até a fase de fruto cereja (ciclo total), de dez cultivares de café (Coffea arabica L.). M édias seguidas de mesma letra no mesmo subperíodo, não diferem entre si a nível de $5 \%$ de probabilidade, pelo teste de Scott-Knott
Pezzopane et al. (2008) verificaram, para uma linhagem não determinada da cultivar Mundo Novo em Campinas, SP, o valor de $761 \mathrm{~mm}$ de evapotranspiração acumulada contra $561 \mathrm{~mm}$ verificada neste trabalho. Contudo, tal discrepância pode estar relacionada com a diferença entre as cultivares uma vez que existem 13 cultivares de Mundo Novo registradas no Ministério da Agricultura, Pecuária e Abastecimento (MAPA, 2009) e os autores não especificaram qual delas estava sendo avaliada. Conforme relatam Pereira et al. (2002), existem diferenças de desenvolvimento entre as linhagens de uma mesma cultivar, o que influencia os valores dos índices bioclimáticos. Não obstante, referida diferença de valores do índice AETR entre este trabalho e aquele citado por Pezzopane et al. (2008), sofreu influência da metodologia de cálculo da evapotranspiração uma vez que esses autores usaram o método proposto por Thonrthwaite (1948), o qual se baseia apenas na temperatura do ar e isto proporciona, frequentemente, superestimativas dos valores da evapotranspiração de referência, o que implicou em valores mais altos do índice baseado na ETo, citados por Pezzopane et al. (2008).

Apesar disto, as demais cultivares apresentaram ciclo tardio, variando o valor de $580 \mathrm{~mm}$ para AETR ou $678{ }^{\circ} \mathrm{C} \mathrm{h}^{-1}$ para IHG, a $616 \mathrm{~mm}$ ou $719^{\circ} \mathrm{C} \mathrm{h}^{-1}$. Essas informações são de grande importância para quem trabalha com a cultura de café, tendo em vista que o escalonamento da colheita otimiza mão-de-obra e máquinas e, com isto, diminuem os custos de produção.

Com relação ao período entre o início da fase de fruto verde cana até a fase de fruto cereja, verificou-se que as cultivares Mundo Novo IAC 379-19 e a Catuaí Vermelho IAC 99 permaneceram mais tempo neste período, quando foram comparadas com as demais cultivares. Com o conhecimento da duração dessa fase é possível programar, de forma racional, a aplicação de adubos, fungicidas e inseticidas (período de carência) e o início da prática de arruação do cafezal.

No que diz respeito ao período que vai do florescimento à fase de fruto verde cana, notou-se que o teste estatístico realizado para os dados do AETR indicou que as cultivares Catuaí Vermelho IAC 99 e Icatu Precoce IAC 3282 diferiram da Mundo Novo IAC 379-19 e Acaiá Cerrado MG 1474, o que não foi constatado quando se utilizaram os dados obtidos pelo IHG. O conhecimento da duração deste subperíodo contribui para a realização racional da adubação e da aplicação de inseticidas contra a broca do café.

\section{CONClusõES}

1. Os índices heliotérmico de Geslin e acúmulo da evapotranspiração de referência, são os mais indicados para a estimativa da duração do ciclo da cultura de café (Coffea arabica L.).

2. Em relação à cultivar Icatu Precoce IAC 3282, considerada padrão, as cultivares foram classificadas em precoce: Acaiá Cerrado MG 1474; intermediária: Mundo Novo IAC 379-19; tardia: Catuaí Vermelho IAC 15, Catuaí Vermelho IAC 99, Catuaí Amarelo IAC 17, Catuaí Amarelo IAC 62, Catuaí Vermelho IAC 144, Rubi MG 1192 e Topázio MG 1190. 


\section{AgradeCIMENTOS}

Os autores agradecem à Fundação de Amparo à Pesquisa do Estado de Minas Gerais - FAPEMIG, pela concessão da bolsa de Pós-Doutorado ao primeiro autor, durante a realização deste trabalho.

\section{LITERATURA CITADA}

Allen, R. G.; Pereira, L. S.; Raes, D.; Smith, M. Crop evapotranspiration: Guidelines for computing crop water requirements. Rome: FAO, 1998. 300p. FAO. Irrigation and Drainage Paper, 56

Assunção, H. F. Relações entre radiação fotossinteticamente ativa e a radiação solar global em Piracicaba, SP. Piracicaba: ESALQ/USP, 1994. 41p. Dissertação Mestrado

Brunini, O.; Lisbão, R. S.; Bernardi, J. B. Temperatura-base para alface "Withe Boston", em um sistema de unidades térmicas. Bragantia, v.35, p.214-219, 1976.

Camargo, M. B. P.; Brunini, O.; Miranda, M. A. C. Modelo agrometeorológico para estimativa da produtividade da cultura da soja no Estado de São Paulo. Bragantia, v.45, p.279-292, 1986.

Lima, E. P.; Silva, E. L. Temperatura base, coeficientes de cultura e graus-dia para cafeeiro arábica em fase de implantação. Revista Brasileira de Engenharia Agrícola e Ambiental, v.12, p.266-273, 2008.
MAPA-Ministério daAgricultura Pecuáriae Abastecimento. Registro Nacional deCultivares. http://masrv103.agricultura.gov.br/proton/ cultivarweb/cultivares_registradas.php?txt_ordem =\&postado $=1 \&$ acao $=$ pesquisar $\&$ first $=C>12$ Out. 2009 .

McMaster, G. S.; Wilhelm, W. W. Growing degree-days: One equation, two interpretations. Agricultural and Forest Meteorology, v.87, p.291-300, 1997.

Pereira, A. R.; Angelocci, L. R.; Sentelhas, P. C. Agrometeorologia: Fundamentos e aplicações práticas. Guaíba: Agropecuária, 2002.478p.

Petek, M. R.; Sera, T.; Fonseca, I. C. B. Exigências climáticas para o desenvolvimento e maturação dos frutos de cultivares de Coffea arabica. Bragantia, v.68, p.169-181, 2009.

Pezzopane, J. R. M.; Pedro Júnior, M. J.; Camargo, M. B. P.; Fazuoli, L. C. Exigência térmica do café arábica cv. Mundo Novo no subperíodo florescimento-colheita. Ciência e Agrotecnologia, v.32, p.1781-1786, 2008.

Sastry, P. S. N.; Chakravarty, N. V. K. Energy summation index for wheat crop in India. Agricultural and Forest Meteorology, v.27, p.45-48, 1982.

Sentelhas, P. C.; Ungaro, M. R. G. Índices bioclimáticos para a cultura de girassol. Scientia Agricola, v.55, p.73-78, 1998.

Thornthwaite, C. W. An approach toward a rational classification of climate. Geographical Review, v.38, p.5594, 1948.

Xue, Q.; Weiss, A.; Baenziger, P. S. Predicting leaf appearance in field grown winter wheat: Evaluating linear and non-linear models. Ecological Modelling, v.175, p.261-270, 2004. 